\title{
Chemical Biology of Sortase A Inhibition: A Gateway to Anti-Infective Therapeutic Agents
}

Rachit Sapra, Amit K. Rajora ${ }^{\dagger}$, Pushpendra Kumar, Govind P. Maurya, Nalin Pant*, V. Haridas*

Department of Chemistry, Indian Institute of Technology Delhi, New Delhi-110016, India.

†Pharmaceutical Sciences Laboratory, Faculty of Science and Engineering, Åbo Akademi University, 20520 Turku, Finland.

*CORRESPONDENCE: haridasv@ chemistry.iitd.ac.in

nalinp@ chemistry.iitd.ac.in

\section{Supporting Information}

\section{Contents:}

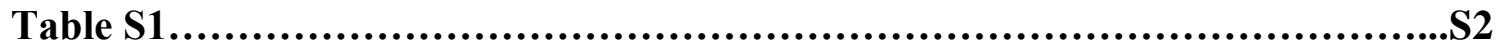

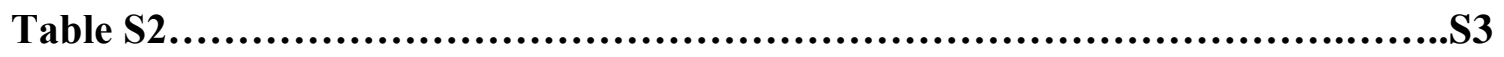

Table S3...................................................................S5

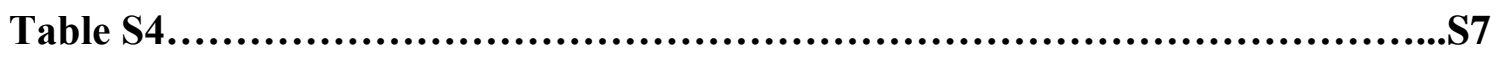


Table S1: Chemical Structures of Selected Peptidomimetic SrtA Inhibitors and Their Binding Parameters

\begin{tabular}{|c|c|c|c|}
\hline Chemical Structure & Binding Parameter & $\begin{array}{c}\text { Assay Used } \\
\text { (Donor-Acceptor) }\end{array}$ & Reference(s) \\
\hline $1 c$ & $44.4^{a}$ & $\begin{array}{c}\text { FRET } \\
\text { (EDANS-Dabcyl) }\end{array}$ & 124 \\
\hline & $10000^{b}$ & $\begin{array}{c}\text { HPLC } \\
\text { (Abz-DNP) }\end{array}$ & 125 \\
\hline
\end{tabular}

${ }^{a}$ Second-order rate constant of inhibition/Inactivation potency $\left(\mathrm{M}^{-1} \mathrm{~min}^{-1}\right)$

${ }^{b} \mathrm{IC}_{50}(\mu \mathrm{M})$ 
Table S2: Chemical Structures of Selected Marine Natural Products as SrtA Inhibitors with Their IC $_{50}$ Values

\begin{tabular}{|c|c|c|c|c|c|}
\hline Chemical Structure & Source & $\begin{array}{c}\text { Class/ } \\
\text { Common Name }\end{array}$ & $\begin{array}{l}\mathrm{IC}_{50} \\
(\mu \mathrm{M})\end{array}$ & $\begin{array}{c}\text { Assay Used } \\
\text { (Donor-Acceptor) } \\
\end{array}$ & Reference(s) \\
\hline 4,5-dihydro & $\begin{array}{l}\text { Spongosorites } \\
\text { sp. }\end{array}$ & $\begin{array}{l}\text { bis(indole) } \\
\text { alkaloid }\end{array}$ & $>245$ & \multirow[t]{4}{*}{$\begin{array}{c}\text { FRET } \\
(\text { EDANS-Dabcyl) }\end{array}$} & 133 \\
\hline $5 e \mathrm{~F}$ & $\begin{array}{l}\text { Spongosorites } \\
\text { sp. }\end{array}$ & $\begin{array}{l}\text { bis(indole) } \\
\text { alkaloid }\end{array}$ & $177.9 \pm 2.5$ & & 133 \\
\hline $5 \mathrm{~g}$ & $\begin{array}{l}\text { Spongosorites } \\
\text { sp. }\end{array}$ & $\begin{array}{l}\text { bis(indole) } \\
\text { alkaloid }\end{array}$ & $>204$ & & 133 \\
\hline & $\begin{array}{l}\text { Spongosorites } \\
\text { sp. }\end{array}$ & $\begin{array}{l}\text { bis(indole) } \\
\text { alkaloid }\end{array}$ & $\begin{array}{c}141.9 \pm 1.4 \\
>244\end{array}$ & & 133 \\
\hline $\begin{array}{l}\text { 10a } R^{1}=B r, R^{2}=H, R^{3}=H, R^{4}=H \\
\text { 10b } R^{1}=H, R^{2}=H, R^{3}=B r, R^{4}=H \\
\text { 10c } R^{1}=B r, R^{2}=H, R^{3}=B r, R^{4}=H \\
\text { 10d } R^{1}=H, R^{2}=H, R^{3}=B r, R^{4}=B r \\
\text { 10e } R^{1}=B r, R^{2}=H, R^{3}=B r, R^{4}=B r \\
\text { 10f } R^{1}=H, R^{2}=B r, R^{3}=B r, R^{4}=B r\end{array}$ & Synoicum sp. & $\begin{array}{c}\text { Eudistomin Y/ } \\
\beta \text {-carboline alkaloids }\end{array}$ & $\begin{array}{c}>272.3 \\
>272.3 \\
163.2 \pm 2.4 \\
146.4 \pm 1.9 \\
>190.5 \\
>190.5\end{array}$ & $\begin{array}{c}\text { FRET } \\
\text { (EDANS-Dabcyl) }\end{array}$ & 139 \\
\hline
\end{tabular}




\begin{tabular}{|c|c|c|c|c|c|}
\hline 14 & $\begin{array}{l}\text { Streptomyces } \\
\text { sp. MBTH32 } \\
\end{array}$ & $\begin{array}{l}\text { Alloxazine/ } \\
\text { Lumichrome }\end{array}$ & $198.2 \pm 0.94$ & $\begin{array}{c}\text { FRET } \\
\text { (EDANS-Dabcyl) }\end{array}$ & 143 \\
\hline 15 & $\begin{array}{c}\text { Aspergillus sp. } \\
\text { F452 }\end{array}$ & $\begin{array}{c}\text { Polyketide/ } \\
\text { Aspermytin A }\end{array}$ & 146 & $\begin{array}{c}\text { FRET } \\
\text { (EDANS-Dabcyl) }\end{array}$ & 144 \\
\hline
\end{tabular}


Table S3: Chemical Structures of Selected Plant-based Natural Products as SrtA Inhibitors with Their IC 50 values

\begin{tabular}{|c|c|c|c|c|c|}
\hline Chemical Structure & Source & Class/Common Name & $\mathrm{IC}_{50}(\boldsymbol{\mu M})$ & $\begin{array}{c}\text { Assay Used } \\
\text { (Donor-Acceptor) }\end{array}$ & References(s) \\
\hline 19c R & Curcuma longa $\mathrm{L}$. & bisdemethoxycurcumin & $103.5 \pm 3.9$ & $\begin{array}{c}\text { FRET } \\
\text { (EDANS-Dabcyl) }\end{array}$ & 148 \\
\hline 20c-Glc & Purchased & Myricitrin & - & Clumping Assay & 162 \\
\hline 21 & $\begin{array}{c}\text { Sorphora } \\
\text { flavescens }\end{array}$ & $\begin{array}{c}\text { Flavanone/ } \\
\text { Kurarinol } \\
\end{array}$ & $107.7 \pm 6.6$ & $\begin{array}{c}\text { FRET } \\
\text { (EDANS-Dabcyl) } \\
\end{array}$ & 163 \\
\hline 22 & Purchased & $\begin{array}{c}O \text {-methylated flavone/ } \\
\text { Acacetin }\end{array}$ & $128.3 \pm 16.5$ & $\begin{array}{c}\text { FRET } \\
\text { (EDANS-Dabcyl) }\end{array}$ & 164 \\
\hline $\begin{array}{l}\text { 29a } \mathrm{R}=\mathrm{H} \\
\text { 29b } \mathrm{R}=\mathrm{CH}_{3}\end{array}$ & Purchased & $\begin{array}{c}\text { Phenylpropanoid/ } \\
\text { Caffeic acid } \\
\text { Ferulic acid }\end{array}$ & $\begin{array}{c}860.4 \\
1323.5\end{array}$ & $\begin{array}{c}\text { FRET } \\
\text { (EDANS-Dabcyl) }\end{array}$ & 171 \\
\hline
\end{tabular}




\begin{tabular}{|c|c|c|c|c|c|}
\hline 31 & Pulsatilla koreana & $\begin{array}{c}\text { Lignan/ } \\
\text { (-)-Rosmarinic acid }\end{array}$ & - & $\begin{array}{c}\text { FRET } \\
\text { (EDANS-Dabcyl) }\end{array}$ & 172 \\
\hline 32 & $\begin{array}{l}\text { Pine Leaves of Cedrus } \\
\text { deodara }\end{array}$ & Phenylpropanoid & 161.02 & $\begin{array}{c}\text { FRET } \\
(\text { EDANS-Dabcyl) }\end{array}$ & 173 \\
\hline
\end{tabular}


Table S4: Chemical Structures of Selected Synthetic Small Molecule SrtA Inhibitors and Their Binding Parameters

\begin{tabular}{|c|c|c|c|c|}
\hline \multirow[b]{2}{*}{ Chemical Structure } & \multicolumn{2}{|c|}{ Binding Parameter } & \multirow[b]{2}{*}{$\begin{array}{c}\text { Assay Used } \\
\text { (Donor-Acceptor) }\end{array}$} & \multirow[b]{2}{*}{ Reference(s) } \\
\hline & $\begin{array}{l}\text { IC }_{50} \\
(\mu \mathrm{M})\end{array}$ & $\begin{array}{l}\text { MIC } \\
(\mu \mathrm{M})\end{array}$ & & \\
\hline $\begin{array}{l}\text { 40a } \mathrm{R}=\mathrm{H} \\
\text { 40b } \mathrm{R}=\mathrm{CF}_{3}\end{array}$ & $\begin{array}{c}736 ; 20.1^{a} \\
190 ; 90^{a}\end{array}$ & $\begin{array}{l}>5950 \\
317.5\end{array}$ & $\begin{array}{c}\text { HPLC } \\
\text { (Abz-DNP) }\end{array}$ & 180 \\
\hline $\begin{array}{l}\text { 41a } \mathrm{R}=\mathrm{CO}_{2} \mathrm{Me} \\
\text { 41b } \mathrm{R}=\mathrm{COOH} \\
\text { 41c } \mathrm{R}=\mathrm{CONH}{ }_{2} \\
\text { 41d } \mathrm{R}=\mathrm{H} \\
\text { 41e } \mathrm{R}=\mathrm{CN}\end{array}$ & $\begin{array}{c}231.09 \pm 6.53 \\
>1000 \\
476.03 \pm 11.08 \\
>1000 \\
187.40 \pm 3.96\end{array}$ & $\begin{array}{l}- \\
- \\
- \\
-\end{array}$ & $\begin{array}{c}\text { FRET } \\
\text { (EDANS-Dabcyl) }\end{array}$ & 181 \\
\hline $41 \mathrm{f}$ & $>1000$ & - & & 181 \\
\hline $\begin{array}{l}\text { 42a } R \\
\text { 42b } R\end{array}$ & $\begin{array}{c}850 \pm 50 ; \\
2.1 \pm 0.12 \times 10^{4 a} \\
1320 \pm 27 ; \\
1.53 \pm 0.26 \times 10^{4 a} \\
1040 \pm 27 ; \\
1.78 \pm 0.21 \times 10^{4 a}\end{array}$ & - & $\begin{array}{c}\text { HPLC } \\
\text { (Abz-DNP) }\end{array}$ & 183 \\
\hline $49 \mathbf{a}$ & $>312$ & - & $\begin{array}{c}\text { FRET } \\
\text { (EDANS-Dabcyl) }\end{array}$ & 191 \\
\hline $\mathbf{5 0 a} \mathrm{R}^{1}=\mathrm{OH}, \mathrm{R}^{2}=\mathrm{COOH}$ & 290 & - & $\begin{array}{c}\text { FRET } \\
\text { (EDANS-Dabcyl) }\end{array}$ & 191 \\
\hline
\end{tabular}




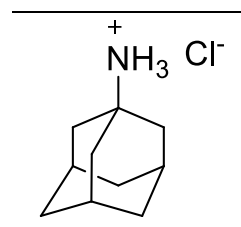

51d

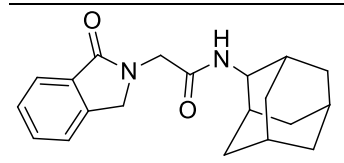

51 e

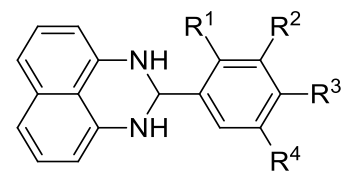

53b $\mathrm{R}^{1,2,3,4}=\mathrm{H}$

53e $\mathrm{R}^{2,4}=\mathrm{H}, \mathrm{R}^{1,3}=\mathrm{Cl}$<smiles>[R]C(=O)C(=NNc1ccccc1)C(=O)O</smiles>

55a $\mathrm{R}^{1}=\mathrm{C}_{2} \mathrm{H}_{5}, \mathrm{R}^{2}=\mathrm{Me}, \mathrm{R}^{3}=\mathrm{H}$
No Inhibition

$>400$

$111 \pm 9$

192

$>270$

$>200$

$>200$

$>200$

$>200$

$>200$

$>200$
$>2663$
FRET

(EDANS-Dabcyl)
192

192

$>1541$

194

FRET and HPLC

(Abz-DNP)<smiles>[R]C(=O)C(=NNc1c([R])cc([R7])c([R7])c1[R])C(=O)O</smiles>

55g $\mathrm{R}^{1}=\mathrm{OH}, \mathrm{R}^{2}=$ 5-methyl-1,2-

oxazole-3-yl, $\mathrm{R}^{3}=\mathrm{R}^{6}=\mathrm{H}, \mathrm{R}^{4}=\mathrm{R}^{5}=\mathrm{Cl}$

FRET

196

55h $\mathrm{R}^{1}=\mathrm{C}_{2} \mathrm{H}_{5}, \mathrm{R}^{2}=1$-ethyl-3-methyl-

$1 \mathrm{H}$-pyrazole-5-yl, $\mathrm{R}^{3}=\mathrm{R}^{6}=\mathrm{H}, \mathrm{R}^{4}=\mathrm{R}^{5}=$

(EDANS-Dabcyl)

55i $\mathrm{R}^{1}=\mathrm{OH}, \mathrm{R}^{2}=1$-ethyl-3-methyl-1H-

pyrazole-5-yl, $\mathrm{R}^{3}=\mathrm{R}^{6}=\mathrm{H}, \mathrm{R}^{4}=\mathrm{R}^{5}=\mathrm{Cl}$

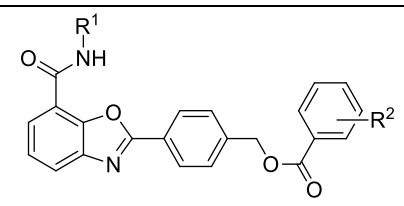

56h $\mathrm{R}^{1}=$ isobutyl, $\mathrm{R}^{2}=4-\mathrm{NO}_{2}$

56i $\mathrm{R}^{1}=$ isopropyl, $\mathrm{R}^{2}=3-\mathrm{OH}$

$158.6 \pm 1.4$

$144.0 \pm 3.1$

$161.3 \pm 1.9$

FRET

197

56j $\mathrm{R}^{1}=n$-Butyl, $\mathrm{R}^{2}=3-\mathrm{OH}$<smiles>O=C(OCc1ccc(-c2nc3ccccc3o2)cc1)c1cccc(O)c1</smiles> 


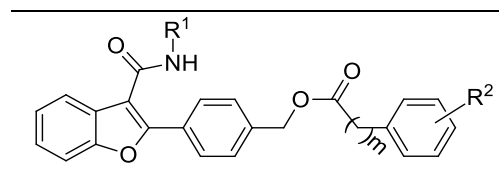

57a $\mathrm{R}^{1}=n$-Butyl, $\mathrm{R}^{2}=4-\mathrm{OH}, \mathrm{m}=0$

$57 \mathrm{~g} \mathrm{R}^{1}=$ isobutyl, $\mathrm{R}^{2}=4-\mathrm{CF}_{3}, \mathrm{~m}=1$

57h $\mathrm{R}^{1}=$ isobutyl, $\mathrm{R}^{2}=4-\mathrm{NO}_{2}, \mathrm{~m}=1$

$186.4 \pm 5.8$

HPLC

198

$57 \mathrm{~m} \mathrm{R}^{1}=$ isobutyl, $\mathrm{R}^{2}=4-\mathrm{F}, \mathrm{m}=0$

$>200$

$>200$

$>200$
(Abz-DNP)
$-$

$-$

${ }^{a}$ Second-order rate constant of inhibition $\left(\mathrm{M}^{-1} \mathrm{~min}^{-1}\right)$ 